\title{
Retrospective Study of the Utility of FLIPI/FLIPI-2 for Follicular Lymphoma Patients Treated with R-CHOP
}

\author{
Ayumi Numata, ${ }^{1)}$ Naoto Tomita, ${ }^{2)}$ Katsumichi Fujimaki, ${ }^{3)}$ Masatsugu Tanaka, ${ }^{1)}$ Chizuko Hashimoto, ${ }^{4)}$ \\ Rika Oshima, ${ }^{5)}$ Kenji Matsumoto, ${ }^{2)}$ Shiro Matsuura, ${ }^{6)}$ Wataru Yamamoto, ${ }^{7)}$ Shigeki Motomura, ${ }^{4)}$ \\ and Yoshiaki Ishigatsubo ${ }^{2}$
}

Keywords: follicular lymphoma, FLIPI/FLIPI-2, R-CHOP

\section{TO THE EDITOR}

Follicular lymphoma (FL) accounts for approximately $20 \%$ of malignant lymphomas. Its clinical course is characterized by a good response to initial treatment, followed by frequent relapses. When the International Prognostic Index ${ }^{1}$ (IPI), which was originally proposed for aggressive lymphoma in 1993, is applied for patients with FL, only a small percentage of patients $(20 \%)$ are classified in the high/highintermediate-risk group. ${ }^{2}$ Therefore, IPI is not fully appropriate in predicting the prognosis of patients with $\mathrm{FL}$ who are considered candidates for intensive or experimental therapy. In 2004, FLIPI $^{2}$ was proposed as a more suitable prognostic index for patients with FL. It consists of 5 adverse prognostic factors: (1) age older than 60 years, (2) advanced clinical stage, (3) hemoglobin level lower than $120 \mathrm{~g} / \mathrm{L}$, (4) more than 4 nodal areas, and (5) elevated serum lactate dehydrogenase level. In 2009, FLIPI-2 ${ }^{3}$ was newly established because FLIPI was found to be inadequate because it was defined before the era of anti-CD20 monoclonal antibody treatments, such as rituximab therapy, and does not include the more

\footnotetext{
Received: December 3, 2011

Revised : January 22, 2012

Accepted : March 6, 2012

1) Department of Hematology, Kanagawa Cancer Center, Yokohama, Japan

2) Department of Internal Medicine and Clinical Immunology, Yokohama City

University Graduate School of Medicine, Yokohama, Japan

${ }^{3)}$ Department of Hematology/Immunology, Fujisawa City Hospital, Fujisawa, Japan

${ }^{4)}$ Department of Medical Oncology, Kanagawa Cancer Center, Yokohama, Japan

${ }^{5)}$ Department of Hematology, Yokohama City University Medical Center, Yokohama, Japan

${ }^{6}$ Department of Hematology, Shizuoka Red Cross Hospital, Shizuoka, Japan

"Department of Hematology, Yamato City Hospital, Yamato, Japan

Address correspondence and reprint requests to : Dr. Naoto Tomita, Department of Internal Medicine and Clinical Immunology, Yokohama City University Graduate School of Medicine, 3-9 Fukuura, Kanazawa, Yokohama 236-0004, Japan

E-mail : cavalier@ch-yamate.dlenet.com
}

recently reported parameters, such as $\beta_{2}$-microglobulin. ${ }^{4}$ FLIPI-2 consists of 5 adverse prognostic factors : (1) elevated $\beta_{2}$-microglobulin, (2) largest node involved having a diameter greater than $6 \mathrm{~cm}$, (3) bone marrow involvement, (4) hemoglobin level lower than $120 \mathrm{~g} / \mathrm{L}$, and (5) age older than 60 years. In establishing the prognostic index of FLIPI-2, the end point was not set as overall survival but as progressionfree survival, reflecting the incurable characteristics of FL. However, the original reported study on FLIPI-2 involved many patients without exposure to rituximab. ${ }^{3}$ Shortly after FLIPI was introduced, one study evaluated the predictive value of FLIPI for patients with advanced-stage FL treated with front-line immunochemotherapy with rituximab plus cyclophosphamide, doxorubicin, vincristine, and prednisone (RCHOP) and proved its usefulness in identifying high-risk patients. ${ }^{5}$ Recently, another study validated the role of FLIPI2 as a prognostic index for patients in the rituximab era. ${ }^{6}$ In this study, we evaluated FLIPI-2 for patients with untreated FL who received initial therapy with R-CHOP.

One hundred and seven patients aged 15 or over with FL who received R-CHOP therapy between 2001 and 2009 were identified from the database of the Yokohama City University Hematology Group. This study was approved by the institutional review board of Kanagawa Cancer Center. The procedures of this study were in accordance with the Helsinki Declaration. Since 2001, the Yokohama City University Hematology Group in Japan has uniformly and curatively treated patients with FL, except those with stage $1 \mathrm{FL}$, with 6 cycles of standard R-CHOP therapy for 21 days. Patients who had partial response (PR) after the 4 initial cycles were administered a total of 8 R-CHOP cycles, whereas patients who did not achieve PR after the 4 initial R-CHOP cycles or those who exhibited disease progression at any given time received salvage therapy. In these cases, the time point to disease progression was determined. Additional local irradia- 
tion was also performed in patients with PR or complete response if deemed necessary by the attending physician. In our 107 patients, the presence or absence of additional radiotherapy was unknown in 2 patients. In the remaining 105 patients, 8 patients (PR in 6 and complete response [CR] in 2) received the additional radiotherapy as first-line therapy. The radiation fields were abdominal nodes in 5, cervical node in 1 , axillar node in 1, and inguinal/femoral node in 1 . However, only $1 \mathrm{CR}$ was newly achieved in the $6 \mathrm{PR}$ patients. No patients received maintenance therapy with rituximab. The presence or absence of a node with a diameter greater than 6 $\mathrm{cm}$ was retrospectively investigated to evaluate the efficacy of FLIPI-2.

The results of patients evaluated by FLIPI and FLIPI-2 are shown in Table 1 in terms of prognostic factors and riskgroup classification. The median age was 57 years (range, 34 to 78). Compared with the patients in the original study on FLIPI- $2,{ }^{3}$ only $10 \%$ of our patients were classified in the lowrisk group because of the high proportion of patients with elevated serum $\beta_{2}$-microglobulin levels $(57 \%)$, with largest node involved having a diameter greater than $6 \mathrm{~cm}(46 \%)$, and with bone marrow involvement $(52 \%)$. There were no correlations between the pathological grades of FL and the risk-group distribution of FLIPI and FLIPI-2 (data not shown). Compared with FLIPI (Fig. 1A), FLIPI-2 (Fig. 1B) was more efficient for identifying three risk groups in terms of progression-free survival using log-rank test; however, the statistical value was marginally significant $(P=0.08)$. The 5year progression-free survival rates according to FLIPI-2

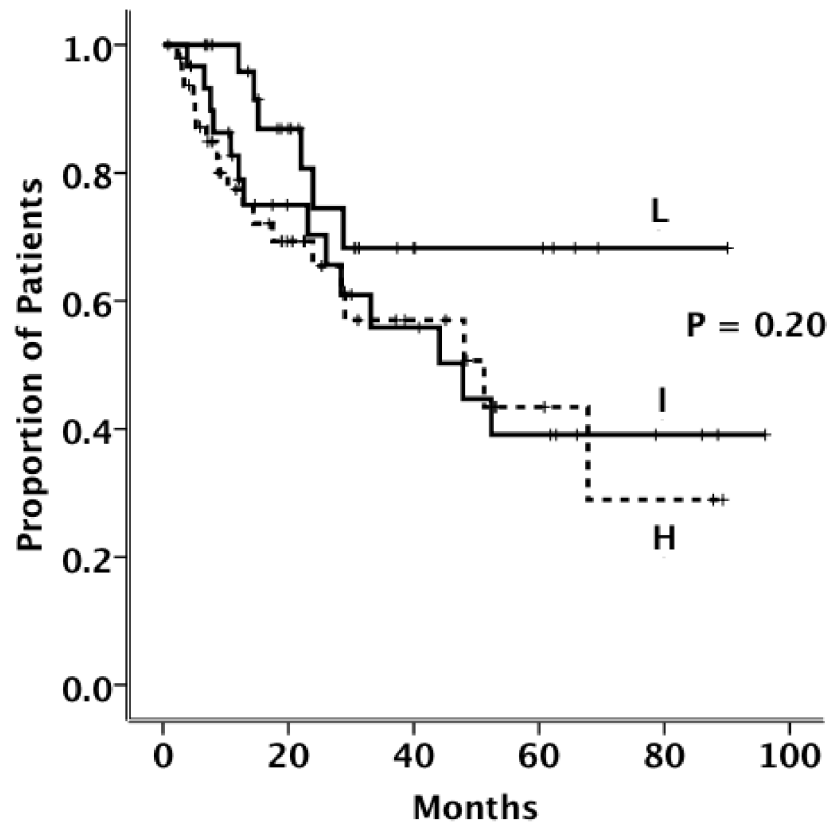

(A)
Table 1. Patient Characteristics

\begin{tabular}{lc}
\hline \multicolumn{1}{c}{ Characteristics } & $(\%)$ \\
\hline Age $>60$ & 42 \\
\hline Male & 49 \\
Elevated LDH & 37 \\
B symptom & 13 \\
PS $\geq 2$ & 7 \\
Stage III, IV & 83 \\
Hb $<120$ g/ L & 19 \\
Elevated $\beta_{2}$-microglobulin & 57 \\
Maximum nodal lesion $>6$ cm & 46 \\
Bone marrow involvement & 52 \\
FLIPI (No. of risk factors) & \\
Low (0-1) & 25 \\
Intermediate (2) & 29 \\
High (3-5) & 46 \\
FLIPI-2 (No. of risk factors) & 10 \\
Low (0) & 49 \\
Intermediate (1-2) & 41 \\
High (3-5) & \\
Pathological grade & 41 \\
1 & 42 \\
2 & 24 \\
3 & \\
\hline LDH, lactate dehydrogenase ; PS, performance \\
status ; Hb, hemoglobin ; FLIPI, Follicular \\
Lymphoma International Prognostic Index
\end{tabular}

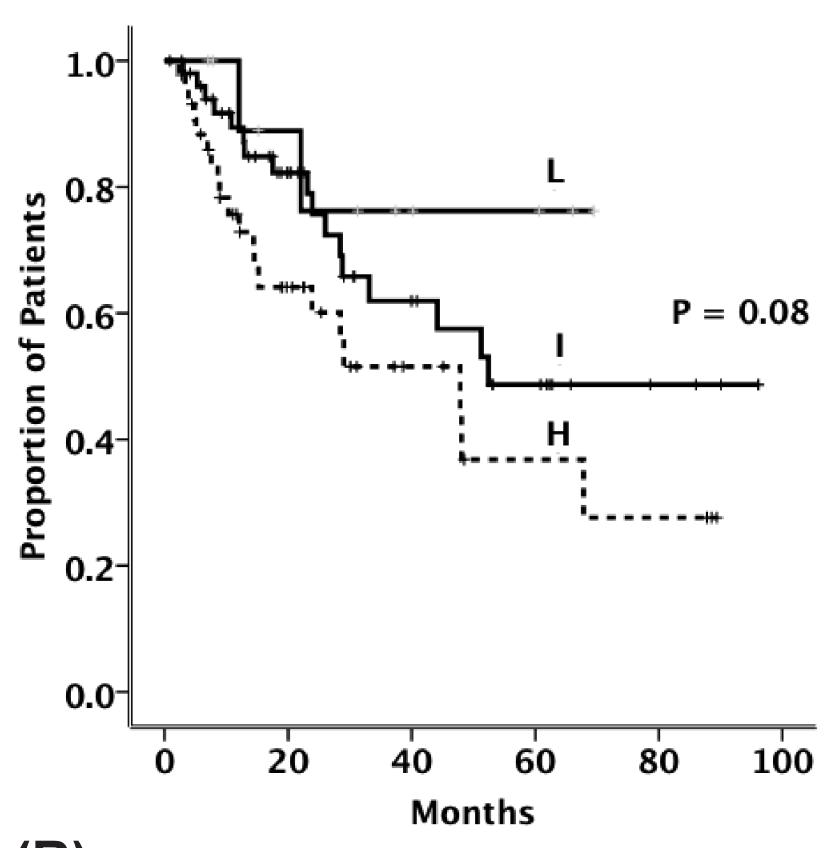

(B)

Fig. 1. Progression-free survival according to FLIPI $(A)$ and FLIPI-2 $(B)$ in 107 patients with follicular lymphoma. 
were $76 \%, 49 \%$, and $37 \%$ in the low-, intermediate-, and high-risk patient groups, respectively. In contrast, FLIPI was somewhat more efficient in predicting overall survival of the patients in the three risk subgroups than FLIPI-2 $(P=0.009$ versus $\mathrm{P}=0.29$, data not shown).

To predict the prognosis of patients with low progressionfree survival rates initially treated with R-CHOP therapy, FLIPI-2 might be more efficient, despite its marginal significance here. It is necessary to validate the efficiency of FLIPI2 by using a large cohort of patients in the rituximab era.

\section{REFERENCES}

1 Project TIN-HsLPF: A predictive model for aggressive nonHodgkin's lymphoma. The International Non-Hodgkin's Lymphoma Prognostic Factors Project. N Engl J Med 329:987994, 1993

2 Solal-Céligny P, Roy P, Colombat P, White J, Armitage JO, et al.: Follicular lymphoma international prognostic index. Blood 104:1258-1265, 2004
3 Federico M, Bellei M, Marcheselli L, Luminari S, LopezGuillermo A, et al.: Follicular lymphoma international prognostic index 2 : a new prognostic index for follicular lymphoma developed by the international follicular lymphoma prognostic factor project. J Clin Oncol 27:4555-4562, 2009

4 Federico M, Guglielmi C, Luminari S, Mammi C, Marcheselli L, et al.: Prognostic relevance of serum $\beta_{2}$-microglobulin in patients with follicular lymphoma treated with anthracycline-containing regimens : A GISL study. Haematologica 92:1482-1488, 2007

5 Buske C, Hoster E, Dreyling M, Hasford J, Unterhalt M, et al.: The Follicular Lymphoma International Prognostic Index (FLIPI) separates high-risk from intermediate- or low-risk patients with advanced-stage follicular lymphoma treated front-line with rituximab and the combination of cyclophosphamide, doxorubicin, vincristine, and prednisone (R-CHOP) with respect to treatment outcome. Blood 108:1504-1508, 2006

6 Arcaini L, Merli M, Passamonti F, Rizzi S, Ferretti V, et al.: Validation of follicular lymphoma international prognostic index 2 (FLIPI2) score in an independent series of follicular lymphoma patients. Br J Haematol 149;455-457, 2010 